\begin{tabular}{|l|l|l||}
\hline \multicolumn{2}{|c|}{ PublisherInfo } \\
\hline \hline PublisherName & $:$ & BioMed Central \\
\hline \hline PublisherLocation & $:$ & London \\
\hline \hline PublisherImprintName & $:$ & BioMed Central \\
\hline \hline
\end{tabular}

\title{
University of Michigan Fibromyalgia Research
}

\begin{tabular}{|l|l|l||}
\hline \multicolumn{2}{|c||}{ ArticleInfo } \\
\hline \hline ArticleID & $:$ & 18 \\
\hline \hline ArticleDOI & $:$ & $10.1186 /$ ar-2000-2-webreport0016 \\
\hline \hline ArticleCitationID & $:$ & 0016 \\
\hline \hline ArticleSequenceNumber & $:$ & 14 \\
\hline \hline ArticleCategory & $:$ & Web Report \\
\hline \hline ArticleFirstPage & $:$ & 1 \\
\hline \hline ArticleLastPage & $:$ & 2 \\
\hline \hline & $:$ & RegistrationDate : 2000-11-21 \\
ArticleHistory & $:$ & OnlineDate \\
\hline \hline ArticleCopyright & $:$ & Current Science Ltd2000-11-21 \\
\hline \hline ArticleGrants & $:$ & \\
\hline \hline ArticleContext & $:$ & 130752211 \\
\hline \hline
\end{tabular}




\section{Overview}

Information about research on fibromyalgia being conducted at the University of Michigan in Ann Arbor is available through this website.

\section{Content}

A webpage provides links to associations, on-line information sources, and supportive resources within Michigan and elsewhere. There is also a link provided on this site's homepage to the University of Michigan Health System Rheumatology Home Page.

\section{References}

1. University of Michigan Fibromyalgia Research. [http://www.med.umich.edu/intmed/rheumatology/ fmweb/] 